\title{
Case Report \\ Guillain-Barré Syndrome due to CMV Reactivation after Cardiac Transplantation
}

\author{
Christina Maria Steger, ${ }^{1}$ Herwig Antretter, ${ }^{2}$ and Daniel Höfer ${ }^{2}$ \\ ${ }^{1}$ Department of Pathology, Innsbruck Medical University, Müllerstraße 44, 6020 Innsbruck, Austria \\ ${ }^{2}$ Department of Cardiac Surgery, Innsbruck Medical University, Anichstrasse 35, 6020 Innsbruck, Austria
}

Correspondence should be addressed to Christina Maria Steger, christina.m.steger@i-med.ac.at

Received 21 December 2011; Accepted 18 January 2012

Academic Editors: O. Amir and G. Minardi

Copyright (C) 2012 Christina Maria Steger et al. This is an open access article distributed under the Creative Commons Attribution License, which permits unrestricted use, distribution, and reproduction in any medium, provided the original work is properly cited.

\begin{abstract}
A 40-year-old male patient suffered from end-stage heart failure due to ischemic cardiomyopathy and received orthotopic cardiac transplantation in June 2005. The instantaneous postoperative course was uneventful, but, seven months later, he suffered from paralysis in the lower extremities finally resulting in quadriplegia and was admitted to hospital. After laboratory testings the diagnosis of a Guillain-Barré syndrome due to cytomegalovirus reactivation was confirmed.
\end{abstract}

\section{Introduction}

Cytomegalovirus (CMV) infection is one of the most important infectious complications of solid-organ transplantation [1] and is responsible for serious, life-threatening diseases in patients infected with human immunodeficiency virus (HIV) [2]. CMV infection, defined as a significant rise in the titre of CMV-specific antibodies, occurs in $44-85 \%$ of kidney, heart, and liver transplant recipients, commonly within the first 3 months after transplantation, when immunosuppression is most intense [3,4]. Cytomegalovirus is a member of the b-herpes-virus group and is characterised by its strict species specificity, long life cycle, and lifetime persistence within the host [5].

Guillain-Barré syndrome (GBS) is a rare acute inflammatory demyelinating polyneuropathy (AIDP) with an incidence of 1 or 2 people per 100,000, an autoimmune disorder affecting the peripheral nervous system, usually triggered by an acute infectious process and exhibiting as an ascending paralysis noted by weakness in the legs that spreads to the upper limbs and the face along with complete loss of deep tendon reflexes. The syndrome was named after the French physicians Guillain, Barré, and Strohl, who were the first to describe it in 1916. It can occur at any age but is most common between ages 30 and 50 and affects both sexes equally. GBS follows viral infection in more than $50 \%$ of cases. The most common antecedent infection is the bacteria Campylobacter jejuni (466\%), followed by Cytomegalovirus (5-15\%), Epstein-Barr virus (2-10\%), Mycoplasma pneumonia (1-5\%), Varicellazoster virus, and acute HIV infection. GBS is also seen in a higher-than-expected rate in patients with sarcoidosis, systemic lupus erythematosus, lymphoma, HIV infection, Lyme disease, and solid tumors, and it may be triggered by mononucleosis, Hodgkin's disease, and rarely rabies or influenza immunizations.

GBS results from an aberrant immune response that somehow mistakenly attacks the nerve tissue of its host, most probably by recognizing a molecular similar epitope mechanism. Circulating antiganglioside antibodies (e.g., GM1, GM2, and GQ1B) found in particular subtypes suggest a molecular mimicry mechanism stimulated by infection. Immune reactions against these epitopes result in acute inflammatory demyelinating neuropathy or acute axonal forms. However, $60 \%$ of cases do not have a known cause; one study suggests that some cases are triggered by the influenza virus or by an immune reaction to the influenza virus.

\section{Case Presentation}

In June 2005, a 40-year-old male patient received orthotopic heart transplantation for end-stage cardiac disease 
secondary to ischemic cardiomyopathy. His past medical history included a myocardial infarction in December 2002 and the implantation of a cardioverter defibrillator in 2003. Preoperative echocardiography showed a grade 3 mitral valve insufficiency, a grade 2 tricuspidal valve insufficiency (NYHA III), and an akinetic anterior ventricular wall with an anterior wall aneurysm. The left ventricular function was highly restricted with an ejection fraction of $30 \%$.

The immediate postoperative course was uneventful. The initial induction protocol after cardiac transplantation consisted of antithymocyte globulin (ATG) for 5 days (total dose $500 \mathrm{mg}$ ), aprednisolone in decreasing dosage, and imurek according to the level of leucocytes. Since day 4 after transplantation cyclosporine A treatment was introduced gradually. For CMV mismatch (CMV-positive recipient and CMV-negative donor) the patient was preemptively treated with Cymevene for 14 days after transplantation. Additionally he received valganciclovir per os since day 15 following transplantation. Postoperatively obtained endomyocardial biopsies were all graded 0R (ISHLT 2004), except one biopsy 3 weeks after transplantation (grade IR).

In January 2006, 7 months after transplantation, he developed an unusual lower-extremity weakness and tingling, finally resulting in quadriplegia and decreased vital capacity requiring mechanical ventilation. Electromyography (EMG) and spinal tap confirmed the diagnosis of GBS (axonal form). CMV early antigen pp65 was positive in polymerase chain reaction and showed 1445 copies CMV/mL, GM1 (IgM, IgG), and GQ1b (IgM, IgG), and Sulfatide IgM antibody levels of the plasma were negative. The patient received 5 cycles of plasmapheresis and Cymevene for 3 weeks. In the following months he achieved nearly full physical recovery.

20 months later, in September 2007, he was admitted to hospital because of pneumonia and received antibiotic treatment. The routinely obtained endomyocardial biopsies revealed a grade IR cellular rejection and the respiratory situation improved, but 10 days after admission he collapsed in the bathroom and died due to sudden cardiac death.

\section{Discussion}

CMV infections still have a substantial impact on graft and patient survival in solid organ transplantation. Threequarters of all patients undergoing solid organ transplantation are believed to experience new infection or reactivation of latent cytomegalovirus (CMV). Of all patients who develop clinical manifestations of CMV infection more than $90 \%$ do so within $1-6$ months after transplantation, and $60 \%$ of the febrile episodes during this period are due to CMV infections. Most of the patients have the so-called self-limiting syndrome, consisting of fever (often spiking), arthralgia, leukopenia and/or thrombocytopenia, and abnormal liver enzymes [6,7].

Aside from this self-limiting syndrome, CMV may cause a myriad of symptoms in the grafted patient: gastrointestinal symptoms including gastrointestinal ulcers, pancreatitis, granulomatous hepatitis, pneumatosis intestinalis, lymphadenopathy, hepatosplenomegaly, pericarditis, myocardi- tis, encephalitis, retinitis, and skin ulcerations associated with vasculitis [6-15].

Risk factors for CMV disease are well recognized and include the following: transplanting a CMV-seronegative patient with a CMV-seropositive donor organ (leading to a primary infection; this is associated with a relative risk of infection >20 times higher than the D-/R-combination). In addition, the recipient's state of immunosuppression plays an important role, determined by the characteristics of the immunosuppressive protocol (type, dose, duration, and timing) as well as various host factors (comorbidity, age, uremia, neutropenia, infections with other immunomodulating viruses, and a high virus load) [6].

As far as the type of immunosuppression is concerned, especially antilymphocyte antibodies and monoclonal antibody preparations such as OKT3 are well known to cause a high incidence of CMV disease when used for either induction or antirejection therapy. And also TNF-a has been suspected to play a role in the reactivation of CMV by stimulation of the CMV major immediate early enhancer/promoter $[16,17]$.

In contrast to AIDS patients, in whom prolonged CMV viremia is the rule, duration of viremia is usually short in transplanted patients, being mostly confined to the period of maximum immunosuppression, that is, shortly after initiation of antirejection therapy. CMV pneumonia after solid organ transplantation is the manifestation that distinguishes serious illness from the more benign disease, and the condition is associated with high mortality, especially when assisted ventilation is required $[6,7,18]$.

GBS has a variety of clinical presentations so that index of suspicion is critical. Typical features are progressive motor weakness and areflexia, often following distal sensory changes. Common presentations include decreased ambulation (or crawling in toddlers), facial weakness, back pain, or sensory changes in the extremities. $85 \%$ have a good recovery; ultimate functional recovery depends on the degree of axonal injury, which can be predicted from electrodiagnostic studies in adults. Early prognosticators include the severity of weakness at the disease nadir and fulminant onset. Overall prognosis in children is better than in adults. Complications include respiratory failure, blood pressure dysregulation (hypotension and/or hypertension), urinary retention, aspiration, pain syndromes, deep venous thrombosis, and infection. Death from early respiratory failure, autonomic instability, or other complications occurs in 3$6 \%$.

Despite numerous reports of GBS in recipients of bone marrow transplants, GBS has rarely been reported in recipients of solid organ transplants. Including the present case, only 4 cases of GBS associated with CMV reactivation after cardiac transplantation are published in the medical literature $[19,20]$ and all of them have much in common (Table 1): all patients were men, all developed GBS within 7 months after transplantation (range 1 week-7 months), all of them received total plasma exchange, and all four patients recovered after illness, although two of them died in the long-term view (one patient died after 13 months because of myocardial infarction, and another one died 
TABLE 1: Reported cases of cytomegalovirus-associated Guillain-Barré syndrome after cardiac transplantation [19].

\begin{tabular}{|c|c|c|c|c|c|c|c|c|c|c|}
\hline Cases (ref) & Sex/age/race & $\begin{array}{l}\text { Transplant } \\
\text { Etiology }\end{array}$ & $\begin{array}{c}\text { CMV } \\
\text { status } \\
\text { Pre-TX } \\
\text { D-R }\end{array}$ & $\begin{array}{c}\text { Evidence } \\
\text { of active } \\
\text { CMV } \\
\text { infection }\end{array}$ & Tx type & $\begin{array}{l}\text { Onset } \\
\text { post-Tx }\end{array}$ & $\begin{array}{l}\text { Ventilator } \\
\text { Support }\end{array}$ & IVIg & TPE & Maximum grade/final grade \\
\hline Baldwin [20] & $\mathrm{M} / 61 / \mathrm{NS}$ & NS & $\mathrm{P}-\mathrm{N}$ & Yes & $\mathrm{OHT}$ & $4 \mathrm{mo}$ & No & Yes & Yes & G4/G1 (died $13 \mathrm{mo}$, acute $\mathrm{MI}$ ) \\
\hline Baldwin [20] & $\mathrm{M} / 62 / \mathrm{C}$ & Rheumatic & $\mathrm{P}-\mathrm{P}$ & Yes & OHT & $4.5 \mathrm{mo}$ & No & Yes & Yes & G4/G2 \\
\hline El-Sabrout [19] & $\mathrm{M} / 62 / \mathrm{B}$ & Dilated CMP & $\mathrm{P}-\mathrm{N}$ & Yes & OHT & $1 \mathrm{wk}$ & Yes & Yes & Yes & G5/G2 \\
\hline Steger & $\mathrm{M} / 40 / \mathrm{C}$ & $\begin{array}{l}\text { Ischemic } \\
\text { CMP }\end{array}$ & $\mathrm{N}-\mathrm{P}$ & Yes & OHT & $6 \mathrm{mo}$ & Yes & No & Yes & $\begin{array}{l}\text { (died } 27 \text { months after Tx, } \\
\text { sudden cardiac death) }\end{array}$ \\
\hline
\end{tabular}

B: black; C: Caucasian; CMP: cardiomyopathy; D: donor; IVIg: intravenous immunoglobulin; MI: myocardial infarction; mo: months; N: negative; NS: not specified; OHT: orthotopic heart transplantation; P: positive; TPE: total plasma exchange; Tx: transplantation; wk: week.

due to sudden cardiac death 27 months after the diagnosis of GBS).

In conclusion, GBS due to CMV reactivation is a rare neurological complication after cardiac transplantation. With prompt treatment by plasmapheresis or intravenous immunoglobulins and supportive care, the majority of patients suffering from GBS will regain full functional capacity. However, death may occur if severe pulmonary complications and autonomic nervous system problems are present.

\section{References}

[1] I. G. Sia and R. Patel, "New strategies for prevention and therapy of cytomegalovirus infection and disease in solidorgan transplant recipients," Clinical Microbiology Reviews, vol. 13, no. 1, pp. 83-121, 2000.

[2] J. S. Dummer, A. Hardy, A. Poorsattar, and M. Ho, "Early infections in kidney, heart, and liver transplant recipients on cyclosporine," Transplantation, vol. 36, no. 3, pp. 259-267, 1983.

[3] R. H. Rubin, S. A. Kemmerly, D. Conti et al., "Prevention of primary cytomegalovirus disease in organ transplant recipients with oral ganciclovir or oral acyclovir prophylaxis," Transplant Infectious Disease, vol. 2, no. 3, pp. 112-117, 2000.

[4] A. Hartmann, S. Sagedal, and J. Hjelmesæth, "The natural course of cytomegalovirus infection and disease in renal transplant recipients," Transplantation, vol. 82, no. 2, pp. S15S17, 2006.

[5] E. S. Mocarski, Cytomegaloviruses and Their Replication, Lippincott-Raven, Philadelphia, Pa, USA, 3rd edition, 1996.

[6] R. H. Rubin, "Infection in the Organ transplant recipient," in Clinical Approach to Infection in the Compromised Host, Y. Rubin, Ed., pp. 629-705, Plenum Press, New York, NY, USA, 1994.

[7] W. J. van Son and T. H. The, "Cytomegalovirus infection after organ transplantation: an update with special emphasis on renal transplantation," Transplant International, vol. 2, no. 3, pp. 147-164, 1989.

[8] M. Ayulo, S. C. Aisner, K. Margolis, and C. Moravec, "Cytomegalovirus-associated gastritis in a compromised host," Journal of the American Medical Association, vol. 243, no. 13 , article $1364,1980$.

[9] E. Foucar, K. Mukai, and K. Foucar, "Colon ulceration in lethal cytomegalovirus infection," American Journal of Clinical Pathology, vol. 76, no. 6, pp. 788-801, 1981.
[10] E. B. Cohen, R. A. Komorowski, H. M. Kauffman, and M. Adams, "Unexpectedly high incidence of cytomegalovirus infection in apparent peptic ulcers in renal transplant recipients," Surgery, vol. 97, no. 5, pp. 606-612, 1985.

[11] B. M. Shrestha, D. Parton, A. Gray et al., "Cytomegalovirus involving gastrointestinal tract in renal transplant recipients," Clinical Transplantation, vol. 10, no. 2, pp. 170-175, 1996.

[12] G. J. Toogood, P. H. Gillespie, S. Gujral et al., "Cytomegalovirus infection and colonic perforation in renal transplant patients," Transplant International, vol. 9, no. 3, pp. 248-251, 1996.

[13] L. Halme, K. Höckerstedt, K. Salmela, and I. Lautenschlager, "CMV infection detected in the upper gastrointestinal tract after liver transplantation," Transplant International, vol. 11, no. 1, pp. S242-S244, 1998.

[14] L. J. Dorfman, "Cytomegalovirus encephalitis in adults," Neurology, vol. 23, no. 2, pp. 136-144, 1973.

[15] N. Minars, J. F. Silverman, M. R. Escobar, and A. J. Martinez, "Fatal cytomegalic inclusion disease: associated skin manifestation in a renal transplant patient," Archives of Dermatology, vol. 113, no. 11, pp. 1569-1571, 1977.

[16] J. Stein, H. D. Volk, C. Liebenthal, D. H. Krüger, and S. Prösch, "Tumour necrosis factoralpha stimulates the activity of the human cytomegalovirus major immediateearly enhancer/promotor in immature monocytic cells," Journal of General Virology, vol. 74, pp. 2333-2338, 1993.

[17] W. D. Döcke, S. Prösch, E. Fietze et al., "Cytomegalovirus reactivation and tumour necrosis factor," The Lancet, vol. 343, no. 8892 , pp. $268-269,1994$.

[18] P. K. Peterson, H. H. Balfour Jr., and S. C. Marker, "Cytomegalovirus disease in renal allograft recipients: a prospective study of the clinical features, risk factors and impact on renal transplantation," Medicine, vol. 59, no. 4, pp. 283-300, 1980.

[19] R. A. El-Sabrout, B. Radovancevic, V. Ankoma-Sey, and C. T. Van Buren, "Guillain-Barré syndrome after solid organ transplantation," Transplantation, vol. 71, no. 9, pp. 13111316, 2001.

[20] R. T. Baldwin, R. R. Pierce, and O. H. Frazier, "Guillain-Barre syndrome after heart transplantation," Journal of Heart and Lung Transplantation, vol. 11, no. 4, pp. 817-819, 1992. 


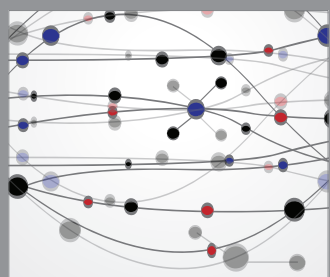

The Scientific World Journal
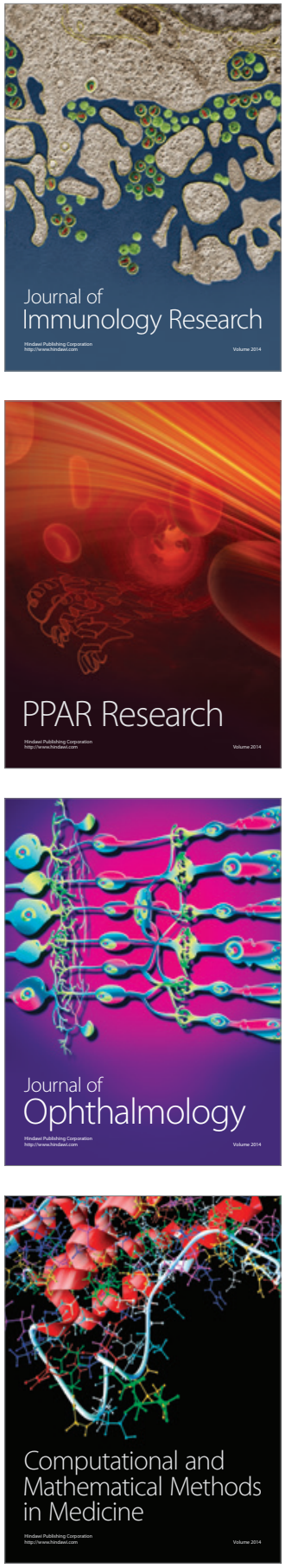



Gastroenterology

Research and Practice


\section{Hindawi}

Submit your manuscripts at

http://www.hindawi.com
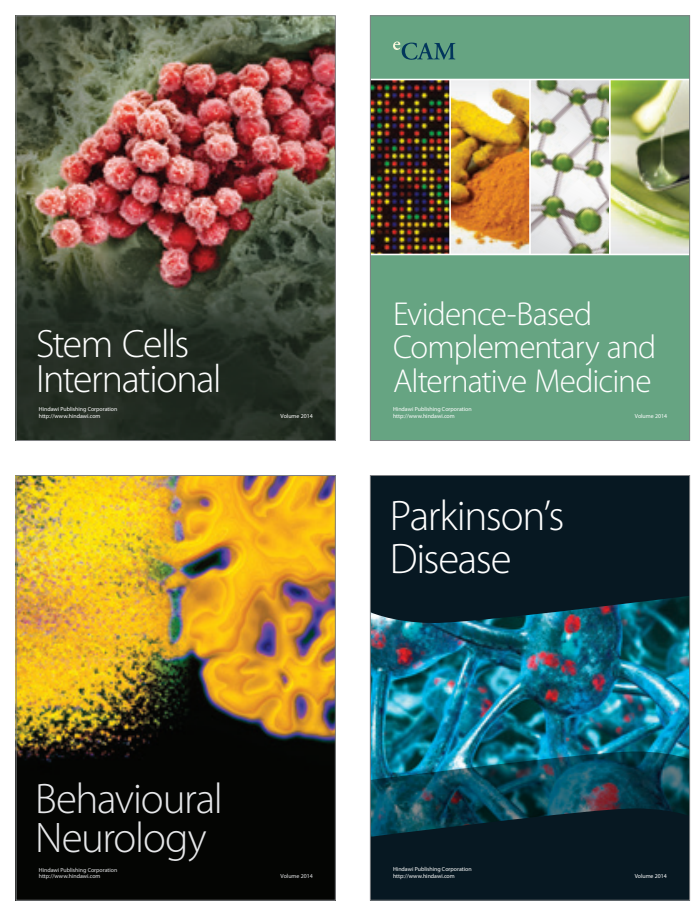

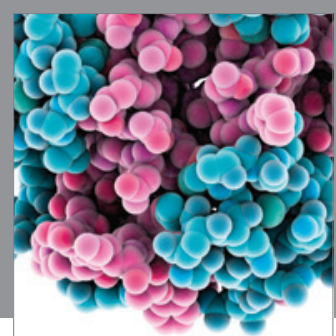

Journal of
Diabetes Research

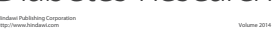



Disease Markers
